\title{
Perfil metabólico de pacientes obesas e não-obesas com a Síndrome dos Ovários
}

\section{Policísticos}

\author{
Metabolic profille of obese and non-obese patients with Polycystic Ovary Syndrome \\ Perfil metabólico de pacientes obesos y no obesos con Síndrome de Ovario Poliquístico
}

Recebido: 10/09/2021 | Revisado: 19/09/2021 | Aceito: 26/09/2021 | Publicado: 27/09/2021

Ione Maria Ribeiro Soares Lopes ORCID: https://orcid.org/0000-0001-9008-3855 Universidade Federal do Piaú, Brasil E-mail:_ione.gin@ @otmail.com

Amanda Nogueira de Castro e Silva ORCID:_https://orcid.org/0000-0002-4958-9886 Universidade Federal do Piaú, Brasil

E-mail:_amandanogueira@outlook.com

\begin{abstract}
Resumo
Avaliar as características clínico-metabólicas de mulheres com Síndrome dos Ovários Policísticos (SOP) de diferentes fenótipos. Realizou-se um delineamento transversal controlado e analítico. A amostragem foi não probabilística e composta por 120 participantes, distribuídas em quatro grupos: 1A (sem SOP e com peso normal); 1B (sem SOP e com peso anormal); 2A (SOP e peso normal) e 2B (SOP e peso anormal). Formulário estruturado e prontuário eletrônico foram utilizados como instrumentos de coleta de dados. Foram realizadas estatísticas uni e bivariadas com o software IBM ${ }^{\circledR}$ SPSS $®$ 21.0. A síndrome metabólica predominou nos grupos $2 \mathrm{~A}(7,1 \%)$ e $2 \mathrm{~B}(37,5 \%)$ em relação aos respectivos controles 1A $(0 \%)$ e 1B $(19,2 \%)$. De forma semelhante, a insulinemia foi maior nos grupos $2 \mathrm{~A}(15,7 \pm 8,2)$ e $2 \mathrm{~B}$ $(19,8 \pm 6,7)$ comparativamente à $1 \mathrm{~A}(6,5 \pm 4)$ e $1 \mathrm{~B}(6,5 \pm 4,8)$. O Teste Oral e Tolerância a Glicose $(75 \mathrm{~g}-2$ horas) foi maior no grupo $2 \mathrm{~B}(132,1 \pm 31,3)$ em relação ao grupo $1 \mathrm{~B}(115,2 \pm 20,8)$ e no grupo $2 \mathrm{~A}(111,9 \pm 24,7)$ em relação ao $1 \mathrm{~A}$ $(105,4 \pm 12,1)$. A pressão arterial sistólica foi superior em $2 \mathrm{~B}(125,8 \pm 11,7)$ em relação à $1 \mathrm{~B}(123,3 \pm 11,7)$ e em $2 \mathrm{~A}$ $(122,1 \pm 10)$ em relação à $1 \mathrm{~A}(113,7 \pm 8,7)$. A SOP se associa a um perfil metabólico desfavorável, em que se sobressaem a resistência insulínica e a síndrome metabólica, tornando essencial um acompanhamento clínico amplo e multiprofissional.
\end{abstract}

Palavras-chave: Síndrome dos ovários policísticos; Resistência à insulina; Síndrome X metabólica; Obesidade.

\begin{abstract}
To evaluate the clinical and metabolic characteristics of women with Polycystic Ovary Syndrome (PCOS) of different phenotypes. Controlled and analytical cross-sectional design. Sampling was non-probabilistic and composed of 120 participants, forming four groups: $1 \mathrm{~A}$ (without PCOS and normal weight); $1 \mathrm{~B}$ (without PCOS and with abnormal weight); 2A (PCOS and normal weight) and 2B (PCOS and abnormal weight). Structured form and electronic medical record were used for data collection. Uni and bivariate statistics were performed using IBM ${ }^{\circledR}$ SPSS ${ }^{\circledR} 21.0$ software. Metabolic syndrome predominated in groups $2 \mathrm{~A}(7.1 \%)$ and $2 \mathrm{~B}(37.5 \%)$ compared to the respective controls $1 \mathrm{~A}(0 \%)$ and 1B $(19.2 \%)$. Similarly, the insulin was higher in groups $2 \mathrm{~A}(15.7 \pm 8.2)$ and $2 \mathrm{~B}(19.8 \pm 6.7)$ compared to $1 \mathrm{~A}(6.5 \pm 4)$ and 1B $(6.5 \pm 4,8)$. The 2-hour oral glucose tolerance test (OGTT) was superior in group 2B $(132.1 \pm 31.3)$ compared to group 1B $(115.2 \pm 20.8)$ and in group 2A $(111.9 \pm 24.7)$ in relation to $1 \mathrm{~A}(105.4 \pm 12.1)$. Total and free testosterone levels were higher in groups $2 \mathrm{~A}(4.4 \pm 6.7$ and $1.4 \pm 1.5$, respectively) and $2 \mathrm{~B}(2 \pm 2.4$ and $1 \pm 0.9)$ in relation to $1 \mathrm{~A}(1,2 \pm 3.8$ and $0.2 \pm 0.3)$ and $1 \mathrm{~B}(0.5 \pm 0.4$ and $0.2 \pm 0.4)$. The systolic blood pressure was higher in $2 \mathrm{~B}(125.8 \pm 11.7)$ than $1 \mathrm{~B}(123.3 \pm 11.7)$ and in $2 \mathrm{~A}(122.1 \pm 10)$ compared to $1 \mathrm{~A}(113.7 \pm 8,7)$. PCOS is associated with an unfavorable metabolic profile, with significance for insulin resistance, hyperandrogenism and metabolic syndrome, making essential the broad and multidisciplinary clinical follow-up.
\end{abstract}

Keywords: Polycystic ovary syndrome; Insulin resistance; Metabolic syndrome X; Obesity.

\section{Resumo}

Evaluar las características clínico-metabólicas de mujeres con síndrome de ovario poliquístico (SOP) de diferentes fenotipos. Se realizó un diseño transversal controlado y analítico. El muestreo fue no probabilístico y estuvo conformado por 120 participantes, distribuidos en cuatro grupos: 1A (sin SOP y con peso normal); 1B (sin síndrome de ovario poliquístico y peso anormal); 2A (SOP y peso normal) y 2B (SOP y peso anormal). Se utilizó el formulario estructurado y la historia clínica electrónica como instrumentos de recolección de datos. Se realizaron estadísticas univariadas y bivariadas utilizando el software IBM® SPSS ${ }^{\circledR}$ 21.0. El síndrome metabólico predominó en los grupos 2A (7,1\%) y 2B (37,5\%) en comparación con los respectivos controles 1A $(0 \%)$ y 1B $(19,2 \%)$. Del mismo modo, la insulinemia fue 
mayor en los grupos $2 \mathrm{~A}(15,7 \pm 8,2)$ y $2 \mathrm{~B}(19,8 \pm 6,7)$ en comparación con $1 \mathrm{~A}(6,5 \pm 4)$ y $1 \mathrm{~B}(6,5 \pm 4,8)$. La prueba oral y la tolerancia a la glucosa $(75 \mathrm{~g}-2$ horas) fue mayor en el grupo $2 \mathrm{~B}(132,1 \pm 31,3)$ en comparación con el grupo $1 \mathrm{~B}$ $(115,2 \pm 20,8)$ y en el grupo $2 \mathrm{~A}(111,9 \pm 24,7)$ en comparación con $1 \mathrm{~A}(105,4 \pm 12,1)$. La presión arterial sistólica fue mayor en $2 \mathrm{~B}(125,8 \pm 11,7)$ en comparación con $1 \mathrm{~B}(123,3 \pm 11,7)$ y en $2 \mathrm{~A}(122,1 \pm 10)$ en comparación con $1 \mathrm{~A}(113,7$ $\pm 8,7)$. El SOP se asocia a un perfil metabólico desfavorable, en el que destacan la resistencia a la insulina y el síndrome metabólico, lo que hace imprescindible un seguimiento clínico amplio y multidisciplinar.

Palabras clave: Síndrome de ovario poliquístico; Resistencia a la insulina; Síndrome de X metabólico; Obesidad.

\section{Introdução}

A Síndrome dos Ovários Policísticos (SOP) é a endocrinopatia mais comum em mulheres no menacme, estando presente em até $18 \%$ dessa população, dependendo dos critérios diagnósticos utilizados. Apesar de tradicionalmente reconhecida pelas repercussões ginecológicas e reprodutivas, associa-se a um cenário clínico mais amplo, heterogêneo e complexo, em que se sobressaem a resistência insulínica, o hiperandrogenismo, a obesidade, a síndrome metabólica e, consequentemente, um maior risco cardiometabólico a longo prazo (Jeanes; Reeves, 2017; Delitala et al., 2017).

Sua etiologia permanece desconhecida, mas acredita-se na origem multifatorial, que envolve alterações da pulsatilidade central do hormônio liberador de gonadotrofinas $(\mathrm{GnRH})$ e do hormônio luteinizante (LH), anormalidades intrínsecas na esteroidogênese ovariana, resistência insulínica, fatores ambientais (dieta e atividade física) e genéticos (pelo menos 16 loci no genoma estariam relacionados à SOP) (Yau et al., 2017).

Em 2003, foi desenvolvido, em Rotterdam, um dos principais consensos para SOP, no qual se diagnostica a síndrome na presença de pelo menos dois dos seguintes critérios: hiperandrogenismo clínico e/ou bioquímico, oligo/anovulação e aspecto policístico dos ovários à ultrassonografia. Posteriormente, em 2009, a Sociedade de SOP e Excesso de Androgênios definiu como critério diagnóstico a presença de hiperandrogenismo clínico e/ou bioquímico, somado à disfunção ovariana e/ou ovários policísticos (Yau et al., 2017).

Cerca de 50 a 70\% das mulheres portadoras de SOP apresentam um comportamento peculiar em relação ao metabolismo dos carboidratos, cursando com resistência insulínica e hiperinsulinemia compensatória, o que predispõe à obesidade. Além disso, a resistência insulínica contribui para o excesso de androgênios, pois estimula a secreção ovariana desses hormônios e reduz a produção hepática de globulina ligadora de hormônios sexuais (SHBG) (Yau et al., 2017).

Nesse contexto de disfunções hormonais e resistência à insulina, a SOP associa-se a fatores de risco cardiovasculares, como obesidade, hipertensão arterial, intolerância à glicose, diabetes, dislipidemia, doença hepática não alcoólica e apneia obstrutiva do sono, com surgimento em idade mais precoce que o habitual. Estima-se que até 35\% da população com SOP apresenta síndrome metabólica (Yau et al., 2017).

A avaliação do perfil metabólico das pacientes diagnosticadas com SOP é uma importante ferramenta capaz de identificar riscos modificáveis para doença cardiovascular e para resultados obstétricos adversos nas mulheres assistidas, permitindo intervenção precoce e melhor prognóstico metabólico e reprodutivo (Delitala et al., 2017).

Desse modo, faz-se necessária uma abordagem clínica ampla e minuciosa dessas pacientes pelos ginecologistas, no sentido de tratar não apenas os distúrbios reprodutivos, mas também de identificar oportunamente aquelas com perfil metabólico de risco, com peso normal e anormal, a fim de instituir tratamento multidisciplinar precoce e minimizar as consequências cardiovasculares a longo prazo (Delitala et al., 2017).

O objetivo geral desse trabalho foi analisar as características clinico-metabólicas de pacientes com síndrome dos ovários policísticos, com diferentes Índices de Massa Corpórea (IMC), a fim de comparar o perfil metabólico das mesmas com mulheres sem SOP, nas mesmas circunstâncias. 


\section{Metodologia}

\section{Desenho de Estudo}

Delineamento transversal controlado e analítico.

\section{Amostra}

A amostragem foi não probabilística, do tipo intencional, e foi composta por 120 participantes, com idade de 18 a 40 anos, atendidas no ambulatório de ginecologia do Hospital Universitário da Universidade Federal do Piauí, no período de julho a dezembro de 2018.

O IMC foi a medida de referência utilizada para a formação dos grupos, considerando peso normal (de 18,5 a 24.9 $\mathrm{kg} / \mathrm{m}^{2}$ ), sobrepeso $\left(\geq 25 \mathrm{e}<30 \mathrm{~kg} / \mathrm{m}^{2}\right)$ e obesidade $\left(\geq 30 \mathrm{~kg} / \mathrm{m}^{2}\right.$ ) (Abeso, 2016).

As participantes foram divididas em 4 grupos: Grupo 1A (controle) - mulheres sem SOP com IMC normal; Grupo 1B (controle) - mulheres sem SOP com sobrepeso/obesidade (IMC anormal); Grupo 2A - mulheres com SOP e IMC normal; Grupo 2B - mulheres com SOP e IMC anormal.

\section{Coleta de dados}

As informações de interesse à pesquisa foram obtidas com as participantes durante a consulta médica, por meio de anamnese, exame físico completo, avaliação de exames complementares e acesso ao prontuário médico-eletrônico.

Todas as pacientes realizaram os exames: glicemia de jejum, teste oral de tolerância à glicose 2 horas após sobrecarga (TOTG 75g), Colesterol Total, Low Density Lipoproteins (LDL), High Density Lipoproteins (HDL), triglicerídeos, hormônio folículo estimulante (FSH), hormônio estimulante da tireóide (TSH), insulina basal, tiroxina livre (T4 livre), hormônio luteinizante (LH), prolactina, testosteronas total e livre, androstenediona, 17-hidroxiprogesterona (17-OH-P), sulfato de dehidroepiandrosterona (S-DHEA) e ultrassonografia pélvica transvaginal ou transabdominal.

Foi utilizado um formulário estruturado pelos pesquisadores, contendo características sociodemográficas, dados antropométricos, estilo de vida, aspectos clínicos e laboratoriais.

\section{Parâmetros clínicos e laboratoriais}

Diagnosticou-se síndrome metabólica pela presença de, no mínimo, três critérios dentre os cinco a seguir: obesidade central (circunferência da cintura superior a $88 \mathrm{~cm}$ ), hipertensão arterial (pressão arterial sistólica $\geq 130$ e/ou pressão arterial diastólica $\geq 85 \mathrm{mmHg}$ ), glicemia alterada (glicemia $\geq 110 \mathrm{mg} / \mathrm{dl}$ ou diagnóstico de diabetes), triglicerídeos $\geq 150 \mathrm{mg} / \mathrm{dl}$ e HDL colesterol $<50 \mathrm{mg} / \mathrm{dl}$ (Sociedade Brasileira de Cardiologia, 2016).

O hiperandrogenismo clínico foi avaliado utilizando a escala modificada de Ferriman e Gallwey, que objetiva avaliar a distribuição de pelos corporais a partir de sua topografia e pigmentação, sendo considerado hirsutismo quando o somatório dos escores estivesse acima de 8 (Flores et al., 2013).

\section{Aspectos éticos}

O estudo foi aprovado pelo Comitê de Ética em Pesquisa do hospital, sob o parecer n. ${ }^{\circ}$ 2.193.433/2017. Todas as participantes assinaram o termo de consentimento livre e esclarecido em duas vias.

\section{Critérios de inclusão}

Mulheres com idade de 18 a 40 anos com diagnóstico de SOP pelos critérios de Rotterdam (2003), e mulheres sem SOP, 
pareadas nas mesmas condições físicas e psicológicas para participar do estudo.

\section{Critérios de exclusão}

Foram excluídas as gestantes, lactantes e puérperas com até 12 semanas pós-parto; pacientes utilizando medicações antiandrogênicas ou de método contraceptivo hormonal há pelo menos 3 meses antes do estudo; portadoras de doenças autoimunes e de endocrinopatias associadas à anovulação e hiperandrogenismo.

\section{Análise dos dados}

Os dados foram processados no software IBM® SPSS ${ }^{\circledR}$, versão 21.0, e foram calculadas estatísticas descritivas, como médias, desvio padrão, mínimos e máximos, para as variáveis quantitativas; e frequências, para as qualitativas. Para as variáveis independentes quantitativas, foi utilizado o Teste de Shapiro-Wilk para verificação da normalidade dos dados e o Teste de Kruskal-Wallis para comparação entre as medidas. Para as variáveis independentes qualitativas, foi utilizado o Teste QuiQuadrado de Pearson e a significância estatística foi estabelecida quando $\mathrm{p}<0,05$.

\section{Resultados}

Ao se aplicarem os critérios de inclusão e exclusão, foi obtida a seguinte amostra: 26 mulheres sem SOP e com peso normal (Grupo 1A), 26 mulheres sem SOP obesas ou com sobrepeso (Grupo 1B), 28 mulheres com SOP e peso normal (Grupo 2A) e 40 mulheres com SOP obesas ou com sobrepeso (Grupo 2B).

A idade das pacientes variou de 18 a 39 anos. As médias das idades foram 29 anos no grupo 2B; 27,9 anos no grupo 2A; 29,1 no grupo $1 \mathrm{~B}$ e 28 anos no grupo $1 \mathrm{~A}$.

Quanto aos dados antropométricos, o IMC médio do grupo 2B foi $29,7 \mathrm{~kg} / \mathrm{m}^{2}$, enquanto no grupo $1 \mathrm{~B}$ foi $29,9 \mathrm{~kg} / \mathrm{m}^{2}$. O IMC médio no grupo $2 \mathrm{~A}$ foi $21,8 \mathrm{~kg} / \mathrm{m}^{2}$, contra $22,9 \mathrm{~kg} / \mathrm{m}^{2}$ no grupo $1 \mathrm{~A}$, com $\mathrm{p}<0,001$. (Tabela 1 )

Em relação à circunferência abdominal, a média no grupo 2B foi de $93,3 \mathrm{~cm}$, enquanto no grupo 1B foi de $84,5 \mathrm{~cm}$. No grupo 2A, foi encontrada uma média de $76,1 \mathrm{~cm}$, contra $71 \mathrm{~cm}$ no grupo $1 \mathrm{~A}$, com $\mathrm{p}<0,001$. (Tabela 1)

$\mathrm{Na}$ avaliação da circunferência do pescoço, a média foi de $35 \mathrm{~cm}$ no grupo 2B, contra $35,6 \mathrm{~cm}$ no grupo 1B; e de 32,4 cm no grupo 2A, contra $32,8 \mathrm{~cm}$ no grupo $1 \mathrm{~A}$, com $\mathrm{p}<0,001$. (Tabela 1 ). 
Tabela 1- Características antropométricas das pacientes, conforme presença de Síndrome dos Ovários Policísticos e índice de massa corporal. Teresina-PI, 2018

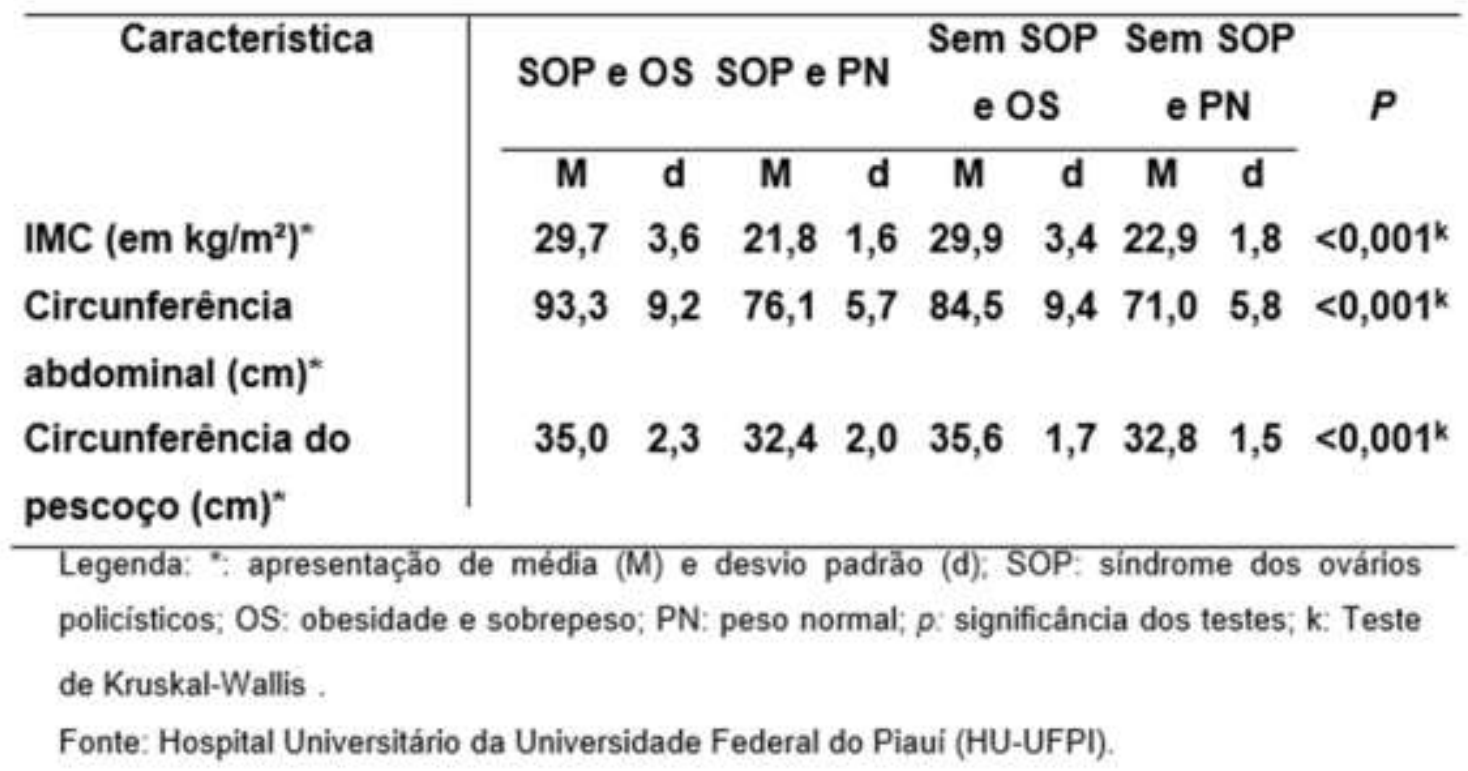

Em relação ao estilo de vida, $95 \%$ das pacientes com SOP obesas não eram etilistas, contra 65,4\% no grupo de obesas sem SOP. Entre as pacientes de peso normal e com SOP, 89,3\% negou etilismo, contra 73,1\% das pacientes de peso normal sem SOP $(\mathrm{p}=0,003)$.

A análise das pacientes com sobrepeso/obesidade em relação ao tabagismo evidenciou que este esteve ausente em $97,5 \%$ das mulheres com SOP e em $84,6 \%$ das pacientes sem SOP. No grupo com SOP e peso normal, $100 \%$ negou tabagismo, contra 92,3\% no grupo controle.

Quanto à prática de atividade física, $80 \%$ das portadoras de SOP obesas afirmou ser adepta, contra 69,2\% das mulheres obesas sem SOP. No grupo com SOP e peso normal, 71,4\% declarou praticar atividade física regularmente, contra $57,7 \%$ das pacientes de peso adequado sem SOP.

Antecedentes familiares patológicos de hipertensão arterial, diabetes, doenças cardiovasculares e/ou dislipidemia estiveram presentes em 62,5\% das mulheres obesas e com SOP, contra 88,5\% naquelas obesas sem SOP. Esse achado positivo na história familiar ocorreu em $85,7 \%$ das pacientes com SOP e peso normal, contra 57,7\% naquelas com peso normal e sem SOP.

O ciclo menstrual no grupo 2B apresentou intervalos com duração média de 63,5(33,6) dias, enquanto no grupo 2A os intervalos duraram em média 59,8(23,4) dias, com p<0,001.

A média da pressão arterial sistólica foi $125,8(11,7) \mathrm{mmHg}$ no grupo $2 \mathrm{~B}$, enquanto no grupo $1 \mathrm{~B}$ foi 123,3(11,7) mmHg. No grupo 2A, a média foi 122,1(10) $\mathrm{mmHg}$, enquanto no grupo $1 \mathrm{~A}$ foi $113,7(8,7) \mathrm{mmHg}, \mathrm{p}$ : 0,008 .

O TOTG 75g 2 horas após a sobrecarga de glicose apresentou média de 132,1(31,3) mg/dl no grupo 2A, de $115,2(20,8) \mathrm{mg} / \mathrm{dl}$ no grupo 1B, de 111,9(24,7) mg/dl no grupo 2A e 105,4(12,1) mg/dl no grupo 1A, p: 0,001.

A insulinemia no grupo 2A teve média de 15,7(8,2) uIU/mL e no grupo $2 \mathrm{~B}$ de $19,8(6,7) \mathrm{uIU} / \mathrm{mL}$, enquanto no controle 1A a média da insulina basal foi de 6,5(4) uIU/mL e no controle $1 \mathrm{~B}$, de $6,5(4,8) \mathrm{uIU} / \mathrm{mL}$, com p $<0,001$ 
(Figura 1).

Figura 1 - Insulinemia basal média das pacientes, conforme presença de Síndrome dos Ovários Policísticos e índice de massa corporal $(n=120)$. Teresina-PI, 2018

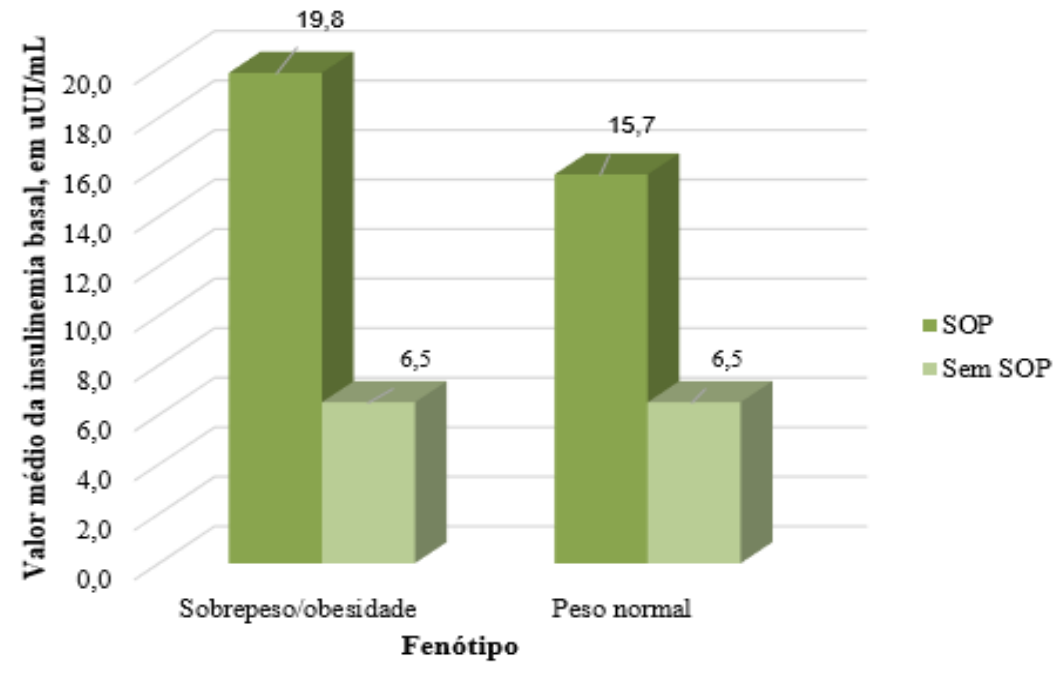

FONTE: Hospital Universitário da Universidade Federal do Piauí

As médias das dosagens de testosteronas total e livre no grupo de peso normal e SOP foram respectivamente $4,4(6,7) \mathrm{ng} / \mathrm{mL}$ e $1,4(1,5) \mathrm{ng} / \mathrm{dL}$, enquanto no grupo peso normal sem SOP foram 1,2(3,8) ng/mL e 0,2(0,3) ng/dL. No grupo de obesas com SOP foram $2(2,4) \mathrm{ng} / \mathrm{mL}$ e $1(0,9) \mathrm{ng} / \mathrm{dL}$, já no grupo de obesas sem SOP foram $0,5(0,4) \mathrm{ng} / \mathrm{mL}$ e $0,2(0,4) \mathrm{ng} / \mathrm{dL}$, com $\mathrm{p}<0,001$ (FIGURA 2).

A média da dosagem de androstenediona no grupo 2B foi $2,4(1,2) \mathrm{ng} / \mathrm{mL}$, no grupo $1 \mathrm{~B}$ foi $0,9(0,6) \mathrm{ng} / \mathrm{mL}$, no grupo 2A foi 1,5(1) ng/mL e, no grupo 1A, foi 1,4(0,9) ng/mL, com $\mathrm{p}<0,001$ (FIGURA 2).

A Síndrome Metabólica foi diagnosticada em 37,5\% das mulheres com SOP e peso anormal, em 19,2\% das pacientes sem SOP com peso anormal e em 7,1\% das pacientes do grupo SOP e peso normal. Não houve nenhum caso no grupo de peso normal sem SOP. Foi constatada Síndrome Metabólica em 25\% das mulheres com SOP nesse estudo e em 9,6\% das mulheres sem a patologia. A obesidade esteve presente em 58,82\% das pacientes com SOP da amostra. 


\section{Figura 2 - Testosteronas total e livre e androstenediona médias das pacientes, conforme presença de Síndrome dos Ovários Policísticos e índice de massa corporal $(n=120)$. Teresina-PI, 2018.}

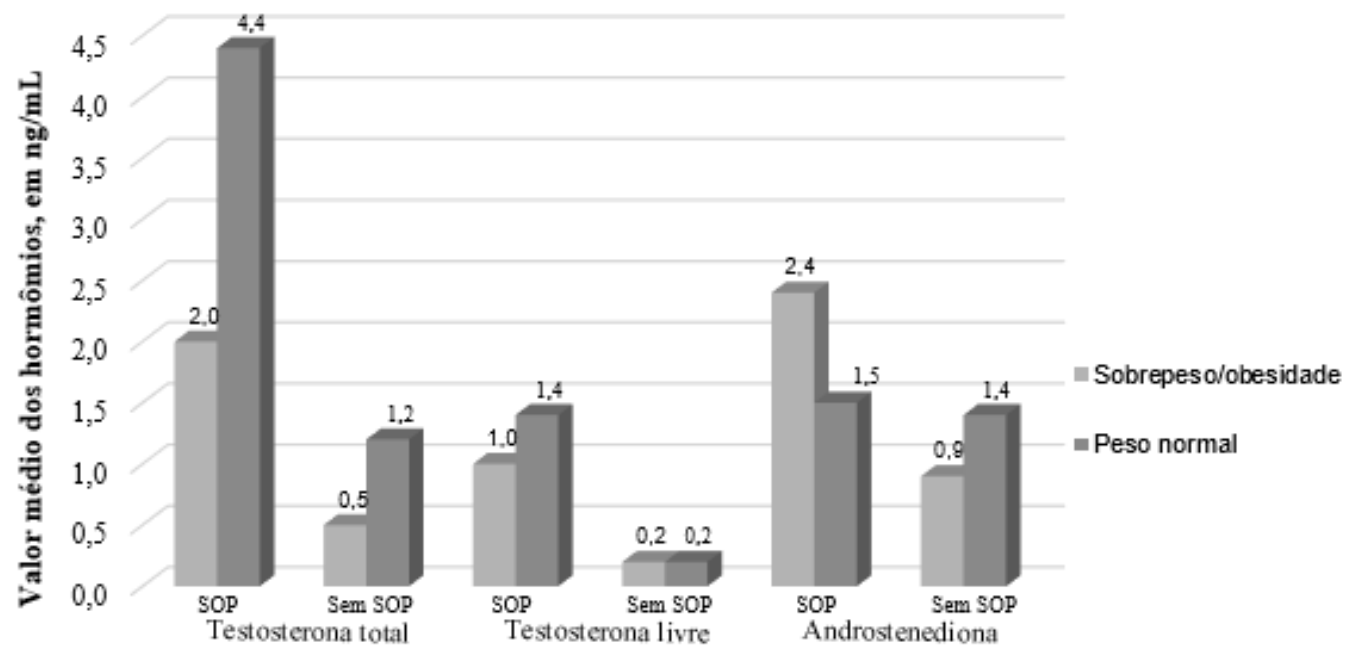

Hormônios e grupos

FONTE: Hospital Universitário da Universidade Federal do Piauí

\section{Discussão}

As participantes do estudo foram mulheres jovens em idade reprodutiva, com idade de 18 a 39 anos. É geralmente nessa faixa etária que o diagnóstico de SOP é realizado, quando as pacientes buscam o ginecologista motivadas pelos distúrbios menstruais vivenciados na síndrome ou encaminhadas da atenção básica, diante do achado ultrassonográfico de ovários micropolicísticos.

Analisando os achados antropométricos, nota-se que apesar do IMC ter sido superior nas pacientes sem SOP em relação às mulheres com SOP de faixa de peso semelhante, a medida da circunferência abdominal foi maior nas pacientes portadoras da síndrome, quando comparadas às de mesma faixa de peso sem SOP. Isso pode ser explicado pelo fato de o IMC não refletir satisfatoriamente a distribuição da gordura corporal, pois uma de suas limitações é não distinguir massa magra de massa gordurosa e a medida da distribuição de gordura é importante na avaliação do risco metabólico, já que a gordura visceral (central) é um fator de risco para doença cardiovascular. Assim, o ideal é que o IMC seja usado em conjunto com medidas da distribuição de gordura, como a medida da circunferência abdominal, que prediz melhor o conteúdo de gordura visceral e também se associa à gordura corporal total (Abeso, 2013).

No contexto da síndrome metabólica, a Circunferência do Pescoço (CP) passou a ser investigada sob o argumento das limitações apresentadas pela medida da circunferência da cintura, como a falta de uniformidade na técnica de mensuração, variações em determinadas condições de saúde e oscilações de medida ao longo do dia. Estudos têm mostrado boa correlação da CP com o IMC, circunferência abdominal, resistência à insulina, níveis de colesterol total, LDL-colesterol e triglicerídeos (Stabe et al., 2013; Küçük et al., 2016).

No presente estudo, a média do CP foi maior nas pacientes sem SOP do que nas pacientes com SOP de mesma faixa de peso, apresentando boa correlação com o IMC, mas não com os demais parâmetros descritos anteriormente, os quais foram preponderantes nos grupos com SOP. 
Em relação ao estilo de vida, observa-se que o tabagismo, o etilismo e o sedentarismo foram menos frequentes nos grupos com SOP em relação aos seus controles sem SOP e de IMC semelhante. Isso pode ser justificado pelo fato de as pacientes sabidamente portadoras de SOP terem sido orientadas sobre a importância de um estilo de vida saudável para o controle das repercussões metabólicas e ginecológicas inerentes à síndrome. De fato, mulheres com SOP e sobrepeso devem ser encorajadas a perder peso, pois uma redução de $5 \%$ já está relacionada a uma melhora dos distúrbios ovulatórios, do hiperandrogenismo clínico/bioquímico e da resistência insulínica (Yau et al., 2016).

História familiar de diabetes é menos comum em pacientes diabéticas com SOP, em relação às diabéticas sem SOP. Isso sugere que a diabetes encontra-se sob diferentes controles genéticos em indivíduos com e sem SOP. Além disso, não se conseguiu, até o momento, identificar uma associação entre os genes conhecidos na hereditariedade do diabetes e a síndrome dos ovários policísticos. A resistência insulínica é o principal fator predisponente para o surgimento de diabetes, mas esse risco aumenta quando ela coexiste com obesidade e história familiar de diabetes nas mulheres com SOP (Delitala et al., 2017; EscobarMorreale, 2017).

A pressão arterial sistólica média, a glicemia 2 horas após TOTG e a insulinemia foram maiores nas pacientes com SOP, quando pareados aos seus controles sem SOP de IMC equivalente. Isso demonstra que o impacto da SOP sobre o metabolismo da glicose independe do excesso de peso, mas é potencializado pela obesidade, com níveis maiores de TOTG $2 \mathrm{~h}$ e insulina basal nas pacientes com SOP obesas do que naquelas com SOP e peso normal (Yau et al., 2016).

A resistência à insulina não é critério diagnóstico de SOP, no entanto, a síndrome parece ter um mecanismo intrínseco de resistência insulínica, fazendo com que níveis maiores de insulina basal possam ser encontrados, qualquer que seja o fenótipo de SOP, em até 50-80\% das pacientes (Jeanes; Reeves, 2017; Delitala et al., 2017).

De acordo com a Androgens Excess and PCOS Society (AE-PCOS), as mulheres com SOP devem ser rastreadas para disfunção metabólica no diagnóstico e em todas as consultas, com avaliação do IMC, circunferência da cintura e pressão arterial. A dislipidemia deve ser rastreada em todas elas a cada 2 anos, obtendo um perfil lipídico completo, e o teste padrão de tolerância à glicose com $75 \mathrm{~g}$ deve ser realizado a cada 2 anos, em mulheres com obesidade e em mulheres não obesas com mais de 40 anos, história pessoal de diabetes gestacional ou história familiar de diabetes tipo 2 (Escobar-Morreale, 2017).

A glicemia de jejum e a hemoglobina glicada não são métodos sensíveis para rastreamento de diabetes em situações de risco como na SOP, uma vez que subdiagnosticam as alterações do metabolismo da glicose nestas pacientes. Estudos mostram que a glicemia de jejum não diagnostica até $80 \%$ dos casos de pré-diabetes e até $50 \%$ dos casos de diabetes nos casos de SOP. Um estudo brasileiro mostrou que o TOTG detectou 5,7\% de DM2, enquanto que a glicemia de jejum detectou 1,2\% de DM2 e $10 \%$ de intolerância à glicose, evidenciando a superioridade e a importância do TOTG em diagnosticar as alterações do metabolismo de glicose nas pacientes com SOP (Pontes; Almeida Filho, 2016).

A análise das dosagens de testosteronas total e livre e androstenediona obtida corrobora com o que é descrito na literatura, já que as pacientes com SOP apresentaram hiperandrogenemia em relação às sem SOP de IMC semelhante.

O hiperandrogenismo é um marcador bioquímico da SOP, presente em até 80-90\% das pacientes e pode ser avaliado através da mensuração de testosteronas total e livre, androstenediona, 17-OH-P, S-DHEA e SHBG. A testosterona livre elevada corresponde à maioria dos achados anormais, apesar de a androstenediona e a S-DHEA também serem bastante utilizadas na prática para o diagnóstico. A S-DHEA tem $80 \%$ da sua produção oriunda das adrenais, enquanto a androstenediona é secretada em quantidade semelhante pelas adrenais e pelas gônadas. Embora os ovários sejam a principal fonte da produção andrógena aumentada na SOP, o excesso de androgênios adrenais pode estar presente em até 25\% dos casos (Delitala et al., 2017).

Ressalta-se que o hiperandrogenismo favorece o depósito de gordura central, que se associa à resistência insulínica e a hiperinsulinemia compensatória, e esta por sua vez atua estimulando a secreção andrógena no ovário, configurando um ciclo 
vicioso de excesso de androgênios e de insulina na circulação e colaborando para a disfunção metabólica (Escobar-Morreale, 2017).

A Síndrome Metabólica (SM) foi encontrada em proporção significativamente maior nas pacientes com SOP do que naquelas de IMC semelhante, mas sem a doença. Destaca-se que houve casos de SM mesmo em pacientes portadoras de SOP sem excesso de peso, enquanto não houve, nessa amostra, nenhum caso em pacientes magras, na ausência de SOP.

Os altos níveis de andrógenos, agravando a adiposidade visceral e perpetuando a resistência insulínica, são os principais moduladores endócrinos da síndrome metabólica associada à SOP. Além disso, mulheres magras com a síndrome, e não apenas as obesas, frequentemente apresentam um perfil lipídico aterogênico, juntamente com outros fatores bioquímicos de risco cardiovascular. Mulheres com SOP têm maior prevalência de SM, enquanto as mulheres com SM frequentemente apresentam SOP (Delitala et al., 2017).

O papel da obesidade central e da resistência insulínica são reconhecidos na gênese da síndrome metabólica, a qual aumenta a mortalidade geral em cerca de 1,5 vezes e a cardiovascular em cerca de 2,5 vezes. Assim, pela sua alta morbidade, ela deve ser diagnosticada e tratada precocemente. Estima-se que o risco de SM em mulheres com SOP seja 2 vezes maior que o das mulheres de idade e IMC semelhantes, sem a doença (Bortoletto et al., 2014; Delitala et al., 2017).

Um estudo transversal, ao avaliar os fatores de risco cardiometabólicos de 295 mulheres chinesas de Hong Kong com SOP e idade média de 30 anos, evidenciou uma prevalência de síndrome metabólica de 24,9\% e um risco 5 vezes maior em relação às mulheres sem SOP, mesmo depois de controlar a idade e o IMC. Em outro estudo envolvendo 170 mulheres asiáticas com SOP, a SM esteve presente em 35,3\% dos participantes (Yau et al., 2017).

Nesse trabalho, a SM foi observada em 25\% das pacientes com SOP e em 9,6\% dos controles, e dentro do grupo SOP, foi mais prevalente entre as obesas. Quando se compara as pacientes de IMC semelhante, a SM predomina nos grupos com SOP (obesas: $37,5 \%$ SOP x 19,2\% sem SOP; não-obesas: 7,1\% SOP x 0\% sem SOP), estando coerente com o que é descrito na literatura (Yau et al., 2017; Delitala et al., 2017).

$\mathrm{Na}$ amostra estudada, 58,82\% das mulheres com SOP apresentavam excesso de peso. Outros autores descrevem que pelo menos metade das pacientes com a doença tem sobrepeso ou obesidade, alcançando uma incidência de até $75 \%$ nos estudos realizados (Yau et al., 2017; Delitala et al., 2017).

A avaliação de resistência à insulina, apesar de atualmente ter valor limitado, pode ser feita através de vários métodos indiretos, dentre eles os métodos Quantitative insulin sensitivity check index (QUICKI) e Homeostasis model assessment Insulin Resistance (HOMA-IR). Valores de insulina de jejum superiores a $12 \mu \mathrm{IU} / \mathrm{mL}$, QUICKI menor que 0,33 e HOMA-IR maior que 2,71 são indicativos de resistência à insulina. Foram obtidas nos grupos SOP deste estudo, médias de insulina de jejum superiores à $12 \mu \mathrm{IU} / \mathrm{mL}$, demonstrando a tendência à resistência à insulina observada na SOP, que não foi encontrada nos grupos controles com IMC semelhante (Pontes; Almeida Filho, 2016).

\section{Considerações Finais}

Os dados obtidos no estudo sugerem que as pacientes com SOP apresentam mais disglicemia, hiperandrogenemia e síndrome metabólica que a população geral de mesma faixa etária e IMC semelhante, mas sem SOP. Com isso, ressalta-se a importância de avaliar o risco metabólico dessas mulheres, identificar subgrupos mais vulneráveis e ofertar tratamento multiprofissional oportuno, aperfeiçoando a assistência à saúde da mulher em relação a essa patologia de grande impacto endócrino, cardiovascular e reprodutivo. 
Research, Society and Development, v. 10, n. 12, e471101220482, 2021

(CC BY 4.0) | ISSN 2525-3409 | DOI: http://dx.doi.org/10.33448/rsd-v10i12.20482

\section{Referências}

Abeso. Associação Brasileira para o Estudo da Obesidade e da Síndrome Metabólica. (2016). Diretrizes brasileiras de obesidade. (4), 1-188.

Bortoletto, M. S. S., Souza, R. K. T. D., Cabrera, M. A. S., \& González, A. D. (2014). Síndrome metabólica em estudos com adultos brasileiros: uma revisão sistemática. Espaç Saúde, 15(4), 86-98.

De Leo, V., Musacchio, M. C., Cappelli, V., Massaro, M. G., Morgante, G., \& Petraglia, F. J. R. B. (2016). Genetic, hormonal and metabolic aspects of PCOS: an update. Reproductive Biology and Endocrinology, 14(1), 1-17.

Delitala, A. P., Capobianco, G., Delitala, G., Cherchi, P. L., \& Dessole, S. (2017). Polycystic ovary syndrome, adipose tissue and metabolic syndrome. Archives of gynecology and obstetrics, 296(3), 405-419.

Escobar-Morreale, H. F. (2017). The role of androgen excess in metabolic dysfunction in women. Sex and Gender Factors Affecting Metabolic Homeostasis, Diabetes and Obesity, 597-608.

Flores, C. B., Flores, L., \& Comim, F. V. (2013). Hirsutismo: avaliação e princípios do tratamento. Revista da AMRIGS, 57(3), 232-9

Jeanes, Y. M., \& Reeves, S. (2017). Metabolic consequences of obesity and insulin resistance in polycystic ovary syndrome: diagnostic and methodological challenges. Nutrition research reviews, 30(1), 97-105.

Küçük, U., Küçük, H. O., Cüce, F., \& Balta, S. (2016). Relationship between neck circumference and epicardial fat thickness in a healthy male population. Arquivos brasileiros de cardiologia, 107, 266-270.

Pontes, A., \& Almeida Filho, B. S. (2016). Síndrome dos ovários policísticos: diagnóstico, tratamento e repercussões ao longo da vida. FMB-UNESP 2016, 97885 .

Sociedade Brasileira de Cardiologia. (2016); VII Diretriz Brasileira de Hipertensão Arterial. Arquivos Brasileiros de Cardiologia, 107,7 -13.

Stabe, C., Vasques, A. C. J., Lima, M. M. O., Tambascia, M. A., Pareja, J. C., Yamanaka, A., \& Geloneze, B. (2013). Neck circumference as a simple tool for identifying the metabolic syndrome and insulin resistance: results from the Brazilian Metabolic Syndrome Study. Clinical endocrinology, 78(6), 874-881. 